\title{
Efficacy of Tart Cherry Juice to Reduce Inflammation Biomarkers among Women with Inflammatory Osteoarthritis (OA)
}

\author{
Kerry S. Kuehl, M.D., Dr.P.H. (Corresponding Author) \\ Dept. of Medicine, Oregon Health \& Science University \\ 3181 SW Sam Jackson Park Rd., Portland, Oregon 97239, United States \\ Tel: 1-503-494-5991_E-mail:kuehlk@ohsu.edu
}

Diane L. Elliot, M.D.

Dept. of Medicine, Oregon Health \& Science University

3181 SW Sam Jackson Park Rd., Portland, Oregon 97239, United States

Tel: 1-503-494-6554 E-mail: elliotd@ohsu.edu

Adriana E. Sleigh, B.S.

Dept. of Medicine, Oregon Health \& Science University

3181 SW Sam Jackson Park Rd., Portland, Oregon 97239, United States

Tel: 1-503-494-3727_E-mail: sleigha@ohsu.edu

Jennifer L. Smith, M.P.H.

Dept. of Medicine, Oregon Health \& Science University

3181 SW Sam Jackson Park Rd., Portland, Oregon 97239, United States

Tel: 1-503-494-3727_E-mail: smjennif@ohsu.edu

Received: July 5, 2012

doi:10.5296/jfs.v1i1.1927
Accepted: July25, 2012 Published: December 1, 2012

URL: http://dx.doi.org/10.5296/jfs.v1i1.1927 


\section{Macrothink}

Journal of Food Studies

ISSN 2166-1073 2012, Vol. 1, No. 1

\section{Abstract}

\section{Background}

Osteoarthritis (OA) is a major cause of pain and disability. OA patients may find relief from the inflammatory component of OA with NSAID use. Tart cherries, high in antioxidant and anti-inflammatory properties, may reduce pain and inflammation without the adverse side effects of NSAIDs. This study aimed to assess the effects of tart cherry juice as compared to a placebo cherry drink on serum biomarkers among inflammatory OA subjects.

\section{Methods}

The design was a randomized, double blind, placebo controlled trial. Twenty inflammatory OA subjects (all female; 40-70 yrs) consumed 10.5 oz bottles of tart cherry juice or placebo cherry drink twice daily for 21 consecutive days. Participants assessed level of pain at baseline and after the intervention. Blood samples were collected at baseline and final visit to assess the biomarkers of inflammation: C-Reactive Protein (CRP), Interleukin-6 (IL-6), Interleukin-10 (IL-10), and Tumor Necrosis Factor-alpha (TMF- $\alpha$ ). Data are reported as mean $+/-$ SD for pre and post serum biomarkers.

\section{Results}

Subjects on the tart cherry juice showed a statistically significant reduction in the serum biomarker CRP $(\mathrm{p}<0.05)$.

\section{Conclusions}

Tart cherry juice may reduce inflammation as measured by certain serum inflammatory biomarkers among women with OA.

Keywords: Inflammatory osteoarthritis, Tart cherry juice, Serum biomarkers 


\section{Introduction}

Epidemiological evidence suggests that a high intake of plant foods is associated with lower risk of chronic diseases. Recently, numerous antioxidant and anti-inflammatory agents have been identified in plants purported to reduce illness and disease associated with inflammation and tissue damage. Specifically, the disease modifying agents include cyclo-oxygenase inhibitory flavonoides (Seeram, Bourquin, \& Nair, 2001; Wang et al., 1999) and anthocynanins with high antioxidant and anti-inflammatory activities (Blando, Gerardi, \& Nicoletti, 2004; Tall et al., 2004). These have been identified in natural foods from black tea to tart cherries to fish oil. Antioxidant and anti-inflammatory agents have been identified in tart cherries, and a study among healthy, non-exercising individuals demonstrated that sweet cherry consumption decreased serum inflammatory biomarkers (Kelley, Rasooly, Jacob, Kader, \& Mackey, 2006). This has led to speculation that consumption of tart cherries may be effective in alleviating symptoms in inflammatory conditions (Tall et al., 2004).

Osteoarthritis (OA) is a common syndrome affecting 65 million Americans characterized by pain and disability (Rayman \& Callaghan, 2006; Felson et al., 2000). Pain relief and improvement of functional disability are the main goals of treatment. A number of studies have looked at dietary factors on inflammation in arthritis patients and some have been purported to improve arthritis pain and function (Pattison et al., 2007). Such natural anti-inflammatory products may be beneficial for the management and treatment of inflammatory diseases without the adverse side effects. Tart cherries have been shown to reduce pain and inflammation in animals and humans (Connolly, McHugh, Padilla-Zakour, Carlson, \& Sayers, 2006; Kuehl, Perrier, Elliot, \& Chesnutt, 2010).

This study was designed to assess the anti-inflammatory effects of tart cherry juice among 40-70 year old inflammatory OA subjects during a randomized, double-blind placebo controlled design. The study's outcomes were to assess changes in serum biomarkers of inflammation.

\section{Materials and Methods}

\subsection{Subjects}

Twenty-one adult subjects aged 40 to 70 years with at least moderate pain from OA, defined as $\geq 40 \mathrm{~mm}$ on a $100-\mathrm{mm}$ pain visual analog scale, were recruited from a panel of individuals with inflammatory OA. All subjects fulfilled the 1990 American College of Rheumatology classification guidelines for the diagnosis of Inflammatory OA (Sayers, Knight, Clarkson, Van Wegen, \& Kamen, 2001). Subjects were in general good health.

Criteria for participation included:

- Ability to maintain same self-directed diet pattern and exercise program for duration of study (three weeks)

- Willingness to maintain stable and consistent pattern of anti-inflammatory or pain relieving drugs use during the course of the study, and to not seek any other treatment during the study 
- Excluded individuals with Type 1 or Type 2 Diabetes

- Excluded individuals who had not been on a stable dose of pain medications or pain modalities for at least three months, including muscle relaxants, tender point anesthetic injections, systemic or intrabursal or intraarticular steroids, or any investigational drug/device in the prior 90 days

- Excluded individuals who used nonphamacologic pain therapies including acupuncture, ultrasound, or transcutaneous electrical nerve stimulation within the past 30 days

Protocol was approved by the institutional review board, and all subjects were provided written informed consent prior to enrollment into the study.

\subsection{Intervention}

Participants consumed two 10.5 oz bottles of either placebo or tart cherry juice daily, with instructions to drink one bottle in the morning and the other in the evening for twenty one consecutive days. The tart cherry juice was tart cultivar Montmorency cherries and one 10.5 oz bottle contained the equivalent of 50-60 cherries. This amount of juice provided at least $600 \mathrm{mg}$ phenolic compounds, expressed as gallic acid equivalents by the method of Singleton and Rossi (Pattison et al., 2007), and at least $40 \mathrm{mg}$ anthocyanins, calculated as cyanidin-3-glucoside equivalents by the $\mathrm{pH}$ differential method described by Giusti and Wrolstad (Pizza, McLoughlin, McGregor, Calomeni, \& Gunning, 2001). The placebo was matched in calorie content, flavor, and consistency to the tart cherry juice. The placebo cherry drink contained $2 \mathrm{~g}$ unsweetened cherry flavored fruit drink mixed with $1 \mathrm{~L}$ water. Cherry syrup and lemon juice were added to match the tart cherry flavor and concentration of soluble solids in the cherry juice blend to a final concentration of 13 Brix (total percentage soluble solids by weight). The flavored beverage was pasteurized and bottled with a guaranteed shelf life of one year. Each bottle had a two-part tear-off label; identification of juice or placebo was concealed, and was only revealed in case of emergency. Treatment assignments were not revealed to study subjects, investigators, clinical staff, or research monitors until all subjects had completed the study and the database had been finalized.

\subsection{Testing Protocol}

A medical history and focused musculoskeletal exam, pain questionnaire, vital signs (sitting pulse and blood pressure), weight and height, and blood test were performed by a medical doctor at the first and final visit. Prior to the first and final visits, subjects completed a three day food diary (DINE) to track nutrient intake. Subjects also completed a satisfaction survey and an adverse effect questionnaire at the final visit.

\subsection{Measurement of Pain and "Pain Scale"}

Pain scores were obtained at the first and final visits using the WOMAC (Western Ontario and McMaster Universities) Index of Osteoarthritis, and using the Visual Analog Scale (VAS) Pain Scale. The WOMAC is a three page questionnaire that assesses pain and quality of life. 


\section{Macrothink}

The VAS Pain Scale is a standardized form where subjects rank current pain on a scale of 1 to 100 .

\subsection{Sample Size and Statistical Analysis}

The sample size for this study was based on the difference in symptoms of the serum inflammation biomarker C-Reactive Protein (CRP). Based on the expected change (SD of the difference) in CRP, it was estimated that, with a sample of 20 subjects, a $14 \%$ difference in CRP could be detected between the placebo and cherry juice trials $(\mathrm{p}<0.05$; power $=80 \%)$. A power analysis was performed using the VAS scale assuming a moderate level of pain among middle-aged female osteoarthritis patients using a ratio of one treated subject for each control subject. Using a placebo controlled study design with a sample size of 10 controls and 10 treated subjects, was an $80 \%$ power to detect a difference in endpoint VAS of -0.28 $($ alpha $=0.05)$. Data were analyzed by independent samples t-test comparison of change score.

\section{Results}

Twenty out of 21 subjects completed the 3-week protocol, one discontinuing the study due to GI upset (nausea and diarrhea) after one week on daily placebo juice (see Figure 1. Biomarkers of inflammation included CRP, Interleukin-6 (IL-6), Interleukin-10 (IL-10), and Tumor Necrosis Factor-alpha (TNF- $\alpha)$. Measures were taken from the participants at the same time of day, in the morning after a 12 hour exercise and food fast, at both baseline and final visit. Table 1 shows subject baseline descriptive data. Despite the placebo group appearing to having an overall lower age and BMI as compared to the active tart cherry juice group, there were no statistically significant differences in subjects at baseline among these characteristics. 


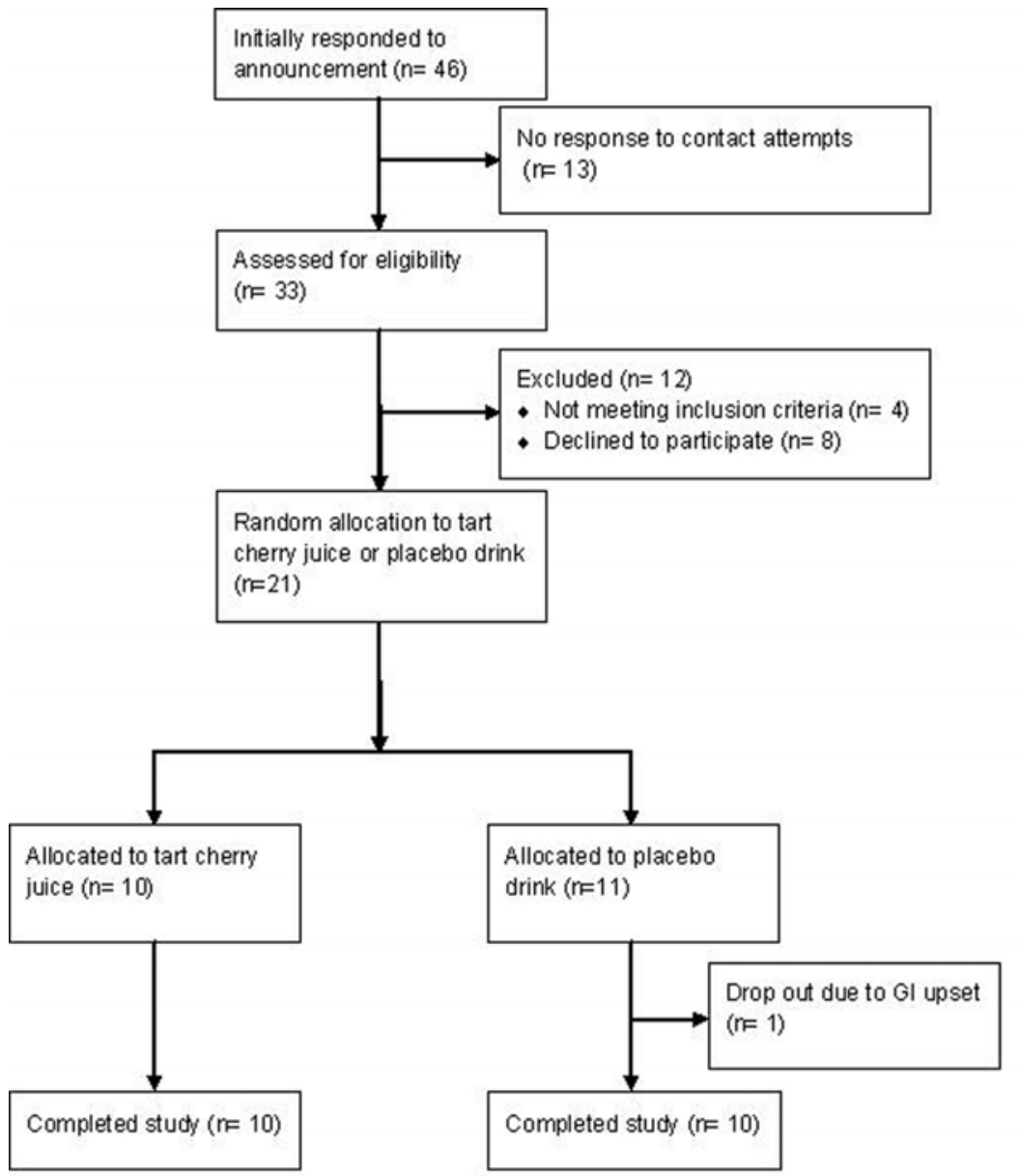

Figure 1. Consort diagram for subject enrollment, randomization, and retention.

Table 1. Baseline characteristics of study participants ( $\mathrm{n}=20$, all female).

\begin{tabular}{|l|l|l|}
\hline & Tart Cherry Juice & Placebo Drink \\
\hline $\mathrm{n}$ & 10 & 10 \\
\hline Age & $55.9 \pm 9.07$ & $52.3 \pm 14.17$ \\
\hline Ethnicity & $\begin{array}{l}\text { Caucasian }(\mathrm{n}=8) \\
\text { Asian }(\mathrm{n}=1) \\
\text { Black/African American }(\mathrm{n}=1)\end{array}$ & $\begin{array}{l}\text { Caucasian }(\mathrm{n}=8) \\
\text { Native Hawaiian/Pacific Islander }(\mathrm{n}=2)\end{array}$ \\
\hline BMI & $31.88 \pm 6.65$ & $27.10 \pm 5.48$ \\
\hline
\end{tabular}

Values are means $\pm \mathrm{SD}$.

Table 2 shows the inflammation biomarker results for the total study group of OA patients. 


\section{Macrothink}

Journal of Food Studies

ISSN 2166-1073

2012, Vol. 1, No. 1

Table 2. Inflammation Biomarkers for total group $(n=20)$, baseline and after 21 days of consumption.

\begin{tabular}{|l|c|c|c|c|}
\hline & $\begin{array}{c}\text { Baseline } \\
\text { (Day 1) }\end{array}$ & $\begin{array}{c}\text { Post-Intervention } \\
\text { (Day 21) }\end{array}$ & Change Score & P value \\
\hline CRP (mg/L) & & & & \\
Tart Cherry (n=10) & $7.19(6.67)$ & $3.77(4.57)$ & $-3.42(4.63)$ & 0.057 \\
Placebo (n=10) & $2.78(3.18)$ & $7.35(12.49)$ & $4.56(11.52)$ & \\
\hline IL-6 (pg/ml) & & & & \\
Tart Cherry (n=10) & $2.44(1.75)$ & $1.89(1.36)$ & $-0.55(1.44)$ & 0.276 \\
Placebo (n=10) & $2.45(3.22)$ & $2.50(2.55)$ & $0.05(0.88)$ & \\
\hline IL-10 (pg/ml) & & & & \\
Tart Cherry (n=10) & $1.23(0.66)$ & $1.32(0.78)$ & $0.01(0.70)$ & 0.858 \\
Placebo (n=10) & $1.03(0.37)$ & $1.18(0.77)$ & $0.15(0.69)$ & \\
\hline TNF- $\boldsymbol{\alpha}(\mathbf{p g} / \mathbf{m l})$ & & & & \\
Tart Cherry (n=10) & $1.62(0.84)$ & $1.39(0.79)$ & $-0.23(0.24)$ & 0.099 \\
Placebo (n=10) & $1.04(0.43)$ & $1.02(0.36)$ & $-0.01(0.31)$ & \\
\hline
\end{tabular}

There were no statistically significant differences between the Tart Cherry and Placebo groups at baseline for all biomarkers.

One subject had an extremely high CRP post test $(41 \mathrm{mg} / \mathrm{L})$ and this sample assay was repeated and found to be the same value. The analysis was performed again without this outlier value, see Table 2 . Results show a statistically significant reduction in CRP in the tart cherry group when this outlier is removed. 
Table 3. Inflammation biomarkers for total group minus outlier $(n=19)$, baseline and after 21 days of consumption.

\begin{tabular}{|c|c|c|c|c|}
\hline & $\begin{array}{l}\text { Baseline } \\
\text { (Day 1) }\end{array}$ & $\begin{array}{c}\text { Post-Intervention } \\
\text { (Day 21) }\end{array}$ & Change Score & $P$ value \\
\hline \multicolumn{5}{|l|}{ CRP (mg/L) } \\
\hline Tart Cherry $(n=10)$ & $7.19(6.67)$ & $3.77(4.57)$ & $-3.42(4.63)$ & \multirow[t]{2}{*}{$0.016^{*}$} \\
\hline Placebo $(n=10)$ & $2.61(3.32)$ & $3.55(3.56)$ & $0.94(1.16)$ & \\
\hline \multicolumn{5}{|l|}{ IL-6 (pg/ml) } \\
\hline Tart Cherry $(n=10)$ & $2.44(1.75)$ & $1.89(1.36)$ & $-0.55(1.44)$ & \multirow[t]{2}{*}{0.364} \\
\hline Placebo $(n=10)$ & $2.51(3.41)$ & $2.47(2.70)$ & $-0.03(0.89)$ & \\
\hline \multicolumn{5}{|l|}{ IL-10 (pg/ml) } \\
\hline Tart Cherry $(n=10)$ & $1.23(0.66)$ & $1.32(0.78)$ & $0.02(0.70)$ & \multirow[t]{2}{*}{0.830} \\
\hline Placebo $(n=10)$ & $1.08(0.35)$ & $1.25(0.79)$ & $0.17(0.73)$ & \\
\hline \multicolumn{5}{|l|}{ TNF- $\alpha(\mathrm{pg} / \mathrm{ml})$} \\
\hline Tart Cherry $(n=10)$ & $1.62(0.84)$ & $1.39(0.79)$ & $-0.23(0.24)$ & \multirow[t]{2}{*}{0.181} \\
\hline Placebo $(n=10)$ & $1.07(0.44)$ & $1.01(0.38)$ & $-0.06(0.29)$ & \\
\hline
\end{tabular}

There were no statistically significant differences between the Tart Cherry and Placebo groups at baseline for all biomarkers. ${ }^{*} \mathrm{p}<0.05$

\section{Discussion}

This study showed a beneficial effect of tart cherry consumption on inflammation. Among subjects consuming the tart cherry juice as compared to the placebo beverage, statistically significant decreases in CRP were observed. Over $40 \%$ of OA has an inflammatory component and the standardization of therapeutic criteria for inflammatory OA has stimulated much research, and understanding of the wide variety of therapeutic approaches is complicated by anecdotal and non-evidenced based basis of OA pain is established (Felson et al., 2000; Adam, 1995). Nonpharmacologic interventions are the mainstay of treatment (patient education, exercise, occupational therapy), but oral medications and nutritional supplements have shown positive and negative results (Fontana et al., 2007; Sundrajun et al., 2004; Pattison et al., 2007). Acetominophen is the mainstay of oral medication treatment of OA pain, but recent studies show that Non-steroidal Anti-inflammatory drugs (NSAIDS) have more benefit on pain and disability due to the anti-inflammatory component which comprised up to $50 \%$ of OA patients in this study. While traditional oral NSAIDs are effective, they can cause serious gastrointestinal, cardiovascular, bleeding, and other adverse events, thus new alternative anti-inflammatory therapies are needed (Felson et al., 2000).

Cyclo-oxygenase inhibitory flavonoids (Seeram et al., 2001; Wang et al., 1999) and anthocynanins with high antioxidant and anti-inflammatory activities (Blando et al., 2004; Tall et al., 2004) have been identified in tart cherries, which are considered good sources of phenolic compounds. This has led to speculation that tart cherry consumption may be 
effective in alleviating symptoms in inflammatory conditions (Tall et al., 2004). Anti-inflammatory drugs and food products containing antioxidant nutrients have been studied extensively in the treatment and prevention of exercise induced muscle damage and its associated symptoms. Some studies have shown efficacy with anti-inflammatory drugs (Donnelly, McCormick, Maughan, Whiting, \& Clarkson, 1988; Dudley et al., 1997; Hasson et al., 1993; Lecomte, Lacroixand, \& Montgomery, 1998; O’Grady et al., 2000; Sayers et al., 2001) whereas others have not (Donnelly, Maughan, \& Whiting, 1990; Howell, Conatser, Chleboun, Karapondo, \& Chila, 1998a; Howell, Conatser, Chleboun, Karapondo, \& Chila, 1998b; Pizza, Cavender, Stockard, Baylies, \& Beighle, 1999). Consumption of about 45 tart cherries a day has been shown to reduce circulating concentrations of inflammatory markers in healthy men and women (Pizza, McLaighlin, McGregor, Calomeni, \& Gunning, 2001; Connolly, McHugh, \& Padilla-Zakour, 2006). Considering the natural anti-inflammatory and antioxidant capacity of tart cherries, it is plausible that tart cherry consumption may have a natural anti-inflammatory effect on healthy and unhealthy individuals.

Recently a study among healthy, exercise naïve individuals demonstrated efficacy for tart cherry juice in decreasing symptoms and strength loss of eccentric exercise induced muscle damage. Most notably, there was a preservation of muscle function attributable to the cherry juice. For the placebo trial, strength loss was $30 \%$ at 24 hours and still $12 \%$ at 96 hours after eccentric exercise. By contrast, in the cherry juice trial, strength loss was only $12 \%$ at 24 hours, and strength was actually 6\% above baseline at 96 hours (Pizza et al., 2001). No studies have been published documenting this effect among OA patients.

Two recent studies published by Kuehl and Elliot showed benefit of tart cherry consumption in fibromyalgia patients and in athletes. Results demonstrated a small but significant reduction in muscle soreness and preservation of muscle strength after a bout of strenuous exercise as compared to placebo drink (Elliot, Kuehl, Dupree Jones, \& Dulacki, 2010). The most recent study assessed the analgesic and myoprotective effects of tart cherry juice among runners participating in a vigorous running event where musculoskeletal injuries and muscle damage occur (Kuehl, Perrier, Elliot, \& Chestnut, 2010). Data showed that drinking tart cherry juice had less musculoskeletal pain as compared to placebo drink when measured on a standardized VAS and there was no difference in adverse events reported between the cherry and placebo juice (Kuehl et al., 2010). Separate research among marathon runners who drank cherry juice five days before, the day of, and after a race showed that those who drank cherry juice had significantly lower levels of specific inflammation markers (Howatson et al., 2010). Tart cherry juice may prevent the symptoms of muscle damage among individuals participating in strenuous exercise.

The specific anti-inflammatory mechanism by which cherry juice supplementation may lessen the damage response is not well understood (Connolly, Sayers, \& McHugh, 2003). The initial damage response of eccentric contractions is a mechanical disruption of myofibrils and injury to the cell membrane. When myofibrillar disruption is extensive, this triggers a local inflammatory response that leads to an exacerbation of damage. Leukotrienes increase the vascular permeability, attracting neutrophils to the injury site, resulting in free radical production (Pizza et al., 2001). It is possible that the anti-inflammatory and/or the antioxidant 
effects of cherry juice may mediate this secondary response and avoid the proliferation of myofibrillar disruption. There are no studies directly measuring neutrophil and monocyte activation after eccentric exercise, but this may be a way to understand the mechanism of reducing muscle injury with this natural occurring fruit.

\section{Conclusion}

This study shows the importance of looking at alternative therapies to conventional methods in the treatment and management of osteoarthritis. This study suggests a benefit of tart cherry juice in reducing inflammation as measured by certain serum inflammatory biomarkers among women with OA. This study showed a significant reduction in CRP among all female OA subjects on the tart cherry juice as compared to placebo, once the outlier was removed. OA. A large number of studies have looked at diet on inflammation in arthritis patients and some have been purported to improve OA pain and function (Pattison et al., 2007). As a new approach to the treatment and management of inflammatory OA, tart cherry juice may provide beneficial anti-inflammatory activity helping OA patients manage their disease with less adverse effects than traditional arthritis medications.

Further research is needed to determine the 1) minimum and maximum effect dose of tart cherry consumption and 2) application for other inflammatory disease populations.

\section{Acknowledgements}

This study was supported by the Cherry Research Committee 2009-2010 Biomedical Research Grant and the Oregon Clinical \& Translational Research Institute (OCTRI).

\section{References}

Adam, O. (1995). Anti-inflammatory diet in rheumatic diseases. European Journal of Clinical Nutrition, 49(10), 703-717.

Blando, F., Gerardi, C., \& Nicoletti, I. (2004). Sour cherry (Prunus cerasus L) anthocyanins as ingredients for functional foods. Journal of Biomedicine and Biotechnology, 5, 253-258. http://dx.doi.org/10.1155/S1110724304404136

Connolly, D. A. J., Sayers, S. P., \& McHugh, M. P., (2003). Treatment and prevention of delayed onset muscle soreness. Journal of Strength and Conditioning Research, 17(1), 197-208.

Connolly, D. A., McHugh, M. P., Padilla-Zakour, O. I., Carlson, L., \& Sayers, S. P. (2006). Efficacy of a tart cherry juice blend in preventing the symptoms of muscle damage. British Journal of Sports Medicine, 40(8), 679-683. http://dx.doi.org/10.1136/bjsm.2005.025429

Donnelly, A. E., McCormick, K., Maughan, R. J., Whiting, P. H., \& Clarkson, P. M. (1988). Effects of non-steroidal anti-inflammatory drug on delayed onset muscle soreness and indices of damage. British Journal of Sports Medicine, 22, 35-38. http://dx.doi.org/10.1136/bjsm.22.1.35

Donnelly, A. E., Maughan, R. J., \& Whiting, P. H. (1990). Effects of ibuprofen on exercise-induced muscle soreness and indices of muscle damage. British Journal of Sports 
Medicine, 24(3), 191-195. http://dx.doi.org/10.1136/bjsm.24.3.191

Dudley, G. A., Czerkawski, J., Meinrod, A., Gillis, G., Baldwin, A., \& Scarpone, M. (1997). Efficacy of naproxen sodium for exercise-induced dysfunction muscle injury and soreness. Clinical Journal of Sports Medicine, 7(1), 3-10. http://dx.doi.org/10.1097/00042752-199701000-00002

Elliot, D.L, Kuehl, K. S., Dupree Jones, K., Dulacki, K. (2010). Using an eccentric exercise-testing protocol to assess the beneficial effects of tart cherry juice in fibromyalgia patients. Integrative Medicine: A Clinician's Journal, 9(6), 25-29.

Felson, D. T., Lawrence, R. C., Dieppe, P. A., Hirsch, R., Jordan, J. M., Kington, R. S., ... Fries, J. F. (2000). Osteoarthritis: New insights. Part 1: The disease and its risk factors. Annals of Internal Medicine, 133(8), 635-646.

Fontana, L., Eagon, J. C., Trujillo, M. E., Scherer, P. E., \& Klein, S. (2007). Visceral fat adipokine secretion is associated with systemic inflammation in obese humans. Diabetes, 56, 1010-1013. http://dx.doi.org/10.2337/db06-1656

Hasson, S. M., Daniels, J. C., Divine, J. G., Niebuhr, B. R., Richmond, S., Stein, P.G., \& Williams, J. H. (1993). Effect of ibuprofen use on muscle soreness, damage, and performance: A preliminary study. Medicine \& Science in Sports \& Exercise, 25(1), 9-17. http://dx.doi.org/10.1249/00005768-199301000-00003

Howatson, G., McHugh, M.P., Hill, J.A., Jewell, A.P., van Someren, K.A., Shave, R.E., \& Howatson, S.A. (2010) Influence of tart cherry juice on indices of recovery following marathon running. Scandinavian Journal of Medicine \& Science in Sports, 20, 843-852. http://dx.doi.org/10.1111/j.1600-0838.2009.01005.x

Howell, J. N., Conatser, R. R., Chleboun, G. S., Karapondo, D. L., \& Chila, A. G. (1998a). The effect of nonsteroidal anti-inflammatory drugs on recovery from exercise-induced muscle injury. 1. Flurbiprofen. Journal of Musculoskeletal Pain, 6(4), 59-68. http://dx.doi.org/10.1300/J094v06n04_06

Howell, J. N., Conatser, R. R., Chleboun, G. S., Karapondo, D. L., \& Chila, A. G. (1998b). The effect of nonsteroidal anti-inflammatory drugs on recovery from exercise-induced muscle injury. 2. Ibuprofen. Journal of Musculoskeletal Pain, 6(4), 69-83. http://dx.doi.org/10.1300/J094v06n04_07

Jacob, R. A., Spinozzi, G. M., Simon, V. A., Kelley, D. S., Prior, R. L., Hess-Pierce, B., \& Kader, A. A. (2003). Consumption of cherries lowers plasma urate in healthy women. Journal of Nutrition, 133, 1826-1829.

Kelley, D. S., Rasooly, R., Jacob, R. A., Kader, A. A., \& Mackey, B. E. (2006) Consumption of bing sweet cherries lowers circulating concentrations of inflammation markers in healthy men and women. American Society of Nutrition, 136(4), 981-986.

Kuehl, K. S., Perrier, E. T., Elliot, D. L., \& Chesnutt, J. C. (2010). Efficacy of tart cherry juice in reducing muscle pain during running: A randomized controlled trial. Journal of the International Society of Sports Nutrition, 7(17). 
Lecomte, J. M., Lacroixand, V. J., \& Montgomery, D. L. (1998). A randomized controlled trial of the effect of naproxen on delayed onset muscle soreness and muscle strength. Clinical $\begin{array}{lllll}\text { Journal of } & \text { Sports } & \text { Medicine, } & \text { 2(2), }\end{array}$ http://dx.doi.org/10.1097/00042752-199804000-00003

O’Grady, M., Hackney, A. C., Schneider, K., Bossen, E., Steinberg, K., Douglas, J. M., Murray, W. J., \& Watkins, W. D. (2000). Diclofenac sodium (Voltaren) reduced exercise-induced injury in human skeletal muscle. Medicine \& Science in Sports \& Exercise, 32(7), 1191-1196. http://dx.doi.org/10.1097/00005768-200007000-00001

Pattison, D. J., Lunt, M., Welch, A., Bingham, S. A., \& Symmons, D. P. M. (2007). Diet and disability in early inflammatory polyarthritis. Rheumatology, 46(Suppl. 1), i122.

Pizza, F. X., Cavender, D., Stockard, A., Baylies, H., \& Beighle, A. (1999). Anti-inflammatory doses of ibuprofen: Effect on neutrophils and exercise-induced muscle injury. International Journal of Sports Medicine, 20(2), 98-102.

Pizza, F. X., McLoughlin, T. J., McGregor, S. J., Calomeni, E. P., \& Gunning, W. T. (2001). Neutrophils injure cultured skeletal myotubes. American Journal of Physiology - Cell Physiology, 281(1), C335-41.

Rayman, M.P., \& Callaghan, A. (2006). Nutrition and arthritis. Blackwell Publishing, Oxford. http://dx.doi.org/10.1002/9780470775011

Sayers, S. P., Knight, C. A., Clarkson, P. M., Van Wegen, E. H., \& Kamen, G. (2001). Effect of ketoprofen on muscle function and sEMG after eccentric exercise. Medicine \& Science in Sports \& Exercise, 33(5), 702-710. http://dx.doi.org/10.1097/00005768-200105000-00004

Seeram, N. P., Bourquin, L. D., \& Nair, M. G. (2001). Degradation products of cyanidin glycosides from tart cherries and their bioactivities. Journal of Agriculture and Food Chemistry, 49(10), 4924-4929. http://dx.doi.org/10.1021/jf0107508

Singleton V. L., \& Rossi, J. A. (1965). Colorimetry of total phenolics with phosphomolybdic-phosphotungstic acid reagent. American Journal of Enology and Viticulture, 16(3), 144-158.

Sundrarjun, T., Komindr, S., Archararit, N., Dahlan, W., Puchaiwatananon, O., Angthararak, S., Udomsuppayakul, U., \& Chuncharunee, S. (2004). Effects of n-3 fatty acids on serum interleukin-6, tumour necrosis factor-alpha and soluble tumour necrosis factor receptor p55 in active rheumatoid arthritis. The Journal of International Medical Research, 32(5), 443-454.

Tall, J. M., Seeram, N. P., Zhao, C., Nair, M. G., Meyer, R. A., \& Raja, S. N. (2004). Tart cherry anthocyanins suppress inflammation-induced pain behavior in rat. Behavioural Brain Research, 15(1), 181-188. http://dx.doi.org/10.1016/j.bbr.2003.11.011

Wang, H., Nair, M. G., Strasburg, G. M., Chang, Y. A., Booren, M., Gray, J. I., \& DeWitt, D. L. (1999). Antioxidant and antiinflammatory activities of anthocyanins and their aglycon, cyanidin, from tart cherries. Journal of Natural Products, 62(2), 294-296. http://dx.doi.org/10.1021/np980501m 